\title{
Development of Electron Optics System for "ETHOS" High-Performance FIB-SEM
}

\author{
Shunsuke Sato ${ }^{1}$, Chisato Kamiya ${ }^{1}$, Ryo Hirano ${ }^{1}$, Tsunenori Nomaguchi ${ }^{1}$, Akinari Morikawa ${ }^{1}$, \\ Hiroyuki Suzuki ${ }^{2}$, Hidekazu Suzuki ${ }^{2}$ and Yo Yamamoto ${ }^{2}$ \\ 1. Science System Product Div., Hitachi High-Technologies corp., Hitachinaka-shi, Japan. \\ 2. Beam Technology Engineering Dep., Hitachi High-Technology Science corp., Oyama-cho, Japan.
}

Thin film specimens of high-performance and leading-edge devices are generally observed by TEM (Transmission Electron Microscope). The thickness, uniformity and the absence of preparationinduced artifact of a lamella can affect how reliable the results are evaluated by TEM. FIB-SEM (Focused Ion Beam-Scanning Electron Microscope) is inevitable for preparing high quality TEM samples. Functions for FIB-SEM such as reducing amorphous damage and detecting the end-point are highly required. The new FIB-SEM [ETHOS NX5000] (Figure 1) enhances the applications by combining a high resolution imaging at low accelerating voltages with a real-time observation function.

The new SEM column (Figure 2) of ETHOS NX5000 is equipped with a magnetic/electrostatic compound lens system as well as a high brightness CFE (Cold Field Emission) electron gun, which is beneficial for observations at low accelerating voltages. The lens system is capable of switching observation modes depending on the purpose or area of interest by HR (High Resolution) mode for high resolution observation or FF (Field-Free) mode for large area observation and other analytical applications (Figure 3).

The ETHOS NX5000 incorporates a new signal detection system which consists of three In-Column detectors that detect various signal electrons with different trajectories depending on electron energy or emission angle. Trajectories of SEs (Secondary Electrons) and high angle BSEs (Back Scattered Electrons) are close to the optical axis due to the magnetic field of lens. Thus, the SEs are detected by the In-Column SE (U) detector and the BSEs are detected by the In-Column BSE (U) detector. The low angle BSEs are detected by the In-Column BSE (L) detector placed at the close end of the inner pole-piece of the objective lens.

Figure 4 shows SEM images of the solder joint area detected by three different types of In-Column detectors and the In-Chamber SE (L) detector. It is possible to display four images taken by these detectors on the PC screen simultaneously (Figure 5). Therefore, users can obtain various information of specimen simultaneously, which helps users to judge the end-point by a real-time observation.

In this report, we give examples of semiconductor device taken with ETHOS and show the characteristic of the In-Column detectors for each lens mode. 


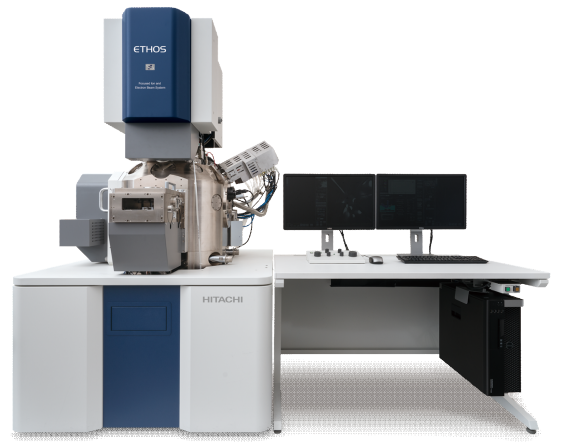

Figure 1. ETHOS NX5000

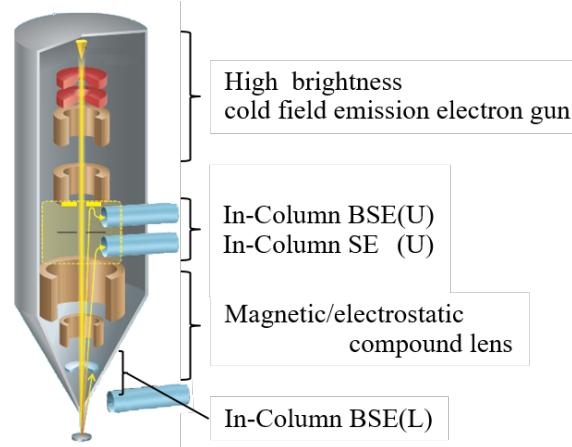

Figure 2. SEM column system

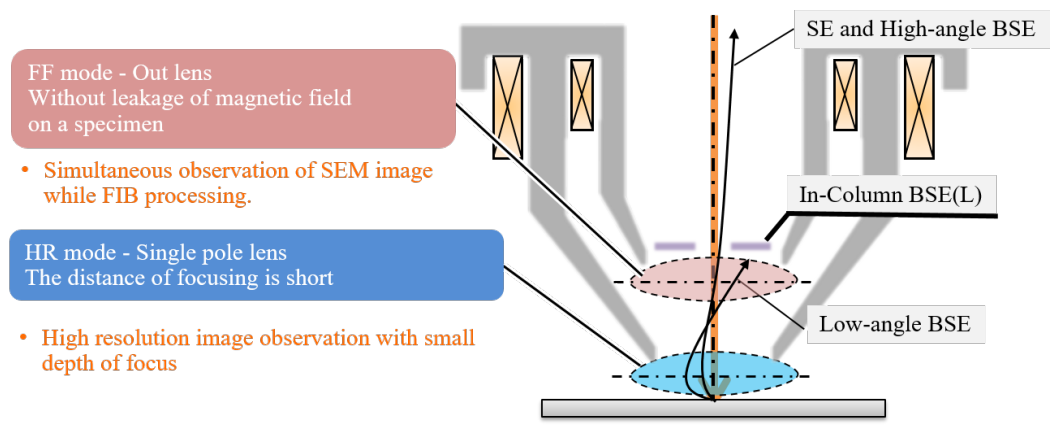

Figure 3. Conceptual objective-lens

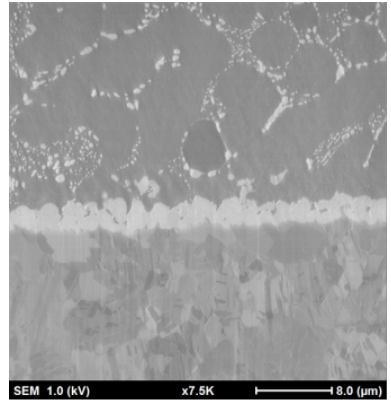

(a) In-Column $\mathrm{SE}(\mathrm{U})$

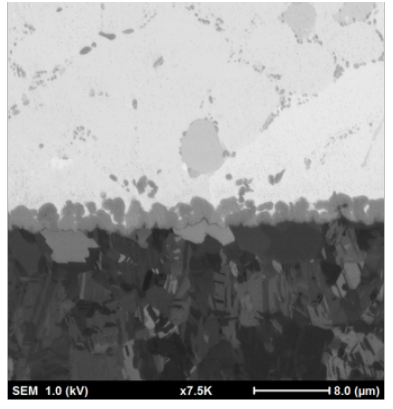

(b) In-Column BSE(U)

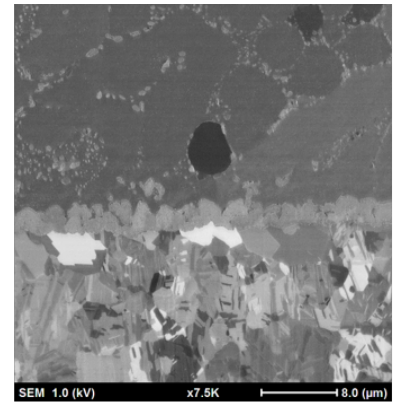

(b) In-Column BSE(L)

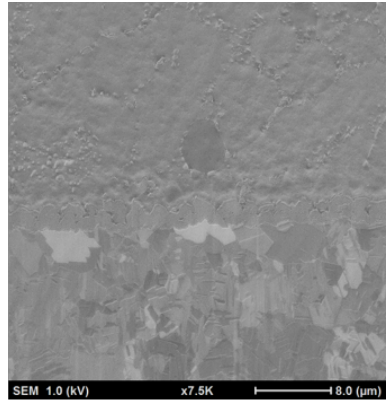

(d) $\mathrm{SE}(\mathrm{L})$

Figure 4. SEM images of the solder joint area

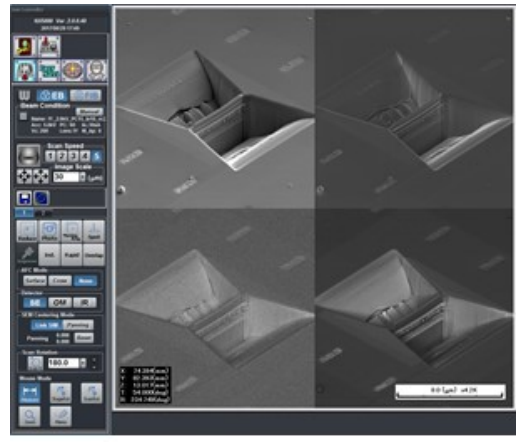

Figure 5. The quad screen with SEM images taken by four detectors 